\title{
One case of toxic epidermal necrolysis after treatment with belimumab in a patient with systemic lupus erythematosus
}

\author{
Madalina Duna', Dinu Valentin Balanescu', Cristina Iosif ${ }^{2}$, Narcis Copca ${ }^{3}$, \\ Denisa Predeteanu', Ruxandra Ionescu ${ }^{1}$ \\ ${ }^{1}$ Department of Internal Medicine and Rheumatology, "Sf. Maria" Clinical Hospital, Bucharest, Romania \\ ${ }^{2}$ Department of Pathology, "Sf. Maria" Clinical Hospital, Bucharest, Romania \\ ${ }^{3}$ Department of Surgery, "Sf. Maria" Clinical Hospital, Bucharest, Romania
}

\begin{abstract}
Belimumab is a human immunoglobulin G1-lambda-1 ( $\operatorname{lgG} 1-\lambda)$ monoclonal antibody that targets the soluble BLyS human protein, also known as B-cell activating factor (BAFF) approved for the treatment of systemic lupus erythematosus (SLE). Serious and sometimes fatal infections have been reported in patients receiving novel immunosuppressive agents, including belimumab. Thus, physicians should exercise caution when considering belimumab in patients with SLE. A 50-year-old woman with SLE presented with a severe, diffuse rash two months after initiating treatment with belimumab. A skin biopsy revealed epidermal necrolysis with keratinocyte detachment and apoptosis in the basal layer of the epidermis, suggestive for toxic epidermal necrolysis (TEN). Belimumab was discontinued and $500 \mathrm{mg}$ of pulse IV methylprednisolone therapy every day for 3 days were administered, with resolution of the skin lesions in the following days. To the best of our knowledge, this is the first case of belimumab-associated TEN.
\end{abstract}

Keywords: systemic lupus erythematosus, autoimmune disease, toxic epidermal necrolysis, belimumab

\section{INTRODUCTION}

Systemic lupus erythematosus (SLE) is a chronic autoimmune disease affecting multiple organs, with a variable severity that can range from mild to lifethreatening (1). People with SLE that receive proper treatment and preventive strategies for complications can have a significantly improved function, disease course, and quality of life (2). The treatment of SLE consists primarily of immunosuppressive drugs, most often hydroxychloroquine and corticosteroids. Both the European Medicines Agency (4) and the United States Food and Drug Administration (FDA) (3) approved belimumab for the treatment of SLE in 2011, thus becoming the first targeted therapy for this disease. Before belimumab, the last drug to be approved by the FDA for the treatment of SLE was hydroxychloroquine in 1955 (5).
Belimumab is a fully humanized $\operatorname{IgG} 1 \gamma$ monoclonal antibody directed against the soluble B lymphocyte stimulator (BLyS) (6). It is the only approved biologic agent for the treatment of SLE. It is indicated as an add-on therapy for the treatment of adult patients with active, autoantibody-positive SLE, who have previously received standard therapy. Belimumab is generally well-tolerated, with common adverse effects including infections, infusion reactions, hypersensitivity, headache, nausea, and fatigue.

We present the case of a 50-year-old woman with SLE who experienced a severe cutaneous adverse reaction two months after initiating treatment with belimumab. 


\section{CASE REPORT}

A 50-year-old woman diagnosed with SLE 2 years prior presented for the sudden onset of a diffuse, scaly, erythematous rash. At the onset of the disease in May 2017, the patient presented with a facial rash and arthritis of the hands. Laboratory findings at that time included hypochromic microcytic anemia, positive inflammatory markers, hypocomplementemia, hyperimmunoglobulinemia, and positive antinuclear antibodies (ANA) and anti-double stranded DNA (anti-dsDNA) antibodies. An ultrasound of the hands revealed synovitis in at least 3 metacarpophalangeal (MCP) joints with grade 2 intra-articular Doppler signal and synovitis of the distal interphalangeal (DIP) joint of the left index finger. The patient met $6 / 16$ points of the revised SLICC criteria (2015) for the SLE diagnosis. Treatment with $400 \mathrm{mg}$ of hydroxychloroquine daily and lowdose (10 mg daily) corticosteroids was initiated. After one month of treatment with hydroxychloroquine, she developed a diffuse rash. Hydroxychloroquine was discontinued and $100 \mathrm{mg}$ of azathioprine (AZA) daily was started. Due to the persistent skin lesions, she received dapsone as consolidation therapy. In December 2018, she presented with papulo-squamous lesions on the face, upper torso, and upper back (Fig. 1a,b). Laboratory tests showed hypochromic microcytic anemia (hemoglobin, $9.5 \mathrm{~g} /$ dl), lymphopenia, hypocomplementemia, and hightiters of ANA and anti-dsDNA antibodies. A 24-hour

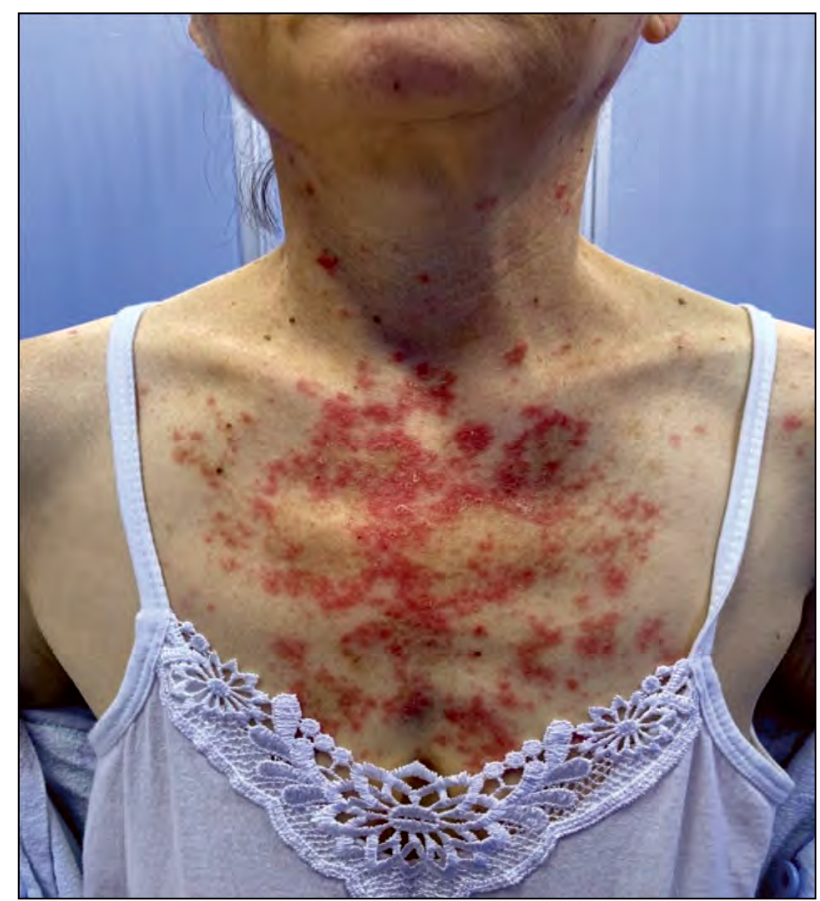

urine protein test was performed to rule out renal involvement and was normal $(754 \mathrm{mg}$ protein/24 hours). An electrocardiogram, abdominal ultrasound and chest X-ray were normal. Transthoracic echocardiography revealed a small amount $(4 \mathrm{~mm})$ of pericardial fluid.

The ultrasound of the hands showed bilateral extensor tenosynovitis (Fig. 2).

Subacute cutaneous lupus was suspected and a skin biopsy was performed. The pathology exam (Fig. 3) showed vacuoles at the level of the basal keratinocytes and perivascular lymphoplasmacytic inflammatory infiltrate, suggestive of subacute SLE. The patient was treated with $250 \mathrm{mg}$ of pulse IV methylprednisolone therapy for four consecutive days, with resolution of the skin lesions. However, the patient had an intense disease activity, with a SELENA-SLEDAI score of 20.

At that moment, the patient met the criteria for therapy with belimumab: age $>18$, a clinical diagnosis of SLE, active disease, positive autoantibodies, stable SLE treatment with glucocorticoids. She received the first 3 loading doses $10 \mathrm{mg} / \mathrm{kg}$ (days 0 , 14,28 ) of belimumab in January 2019, and another dose four weeks later. Following the last infusion, she developed a diffuse erythematous, bullous rash with rapid coalescence and extensive desquamation (Fig. 4a, b) and a positive Nikolsky sign.

Laboratory findings were normal, with the exception of hypocomplementemia, which was not dif-

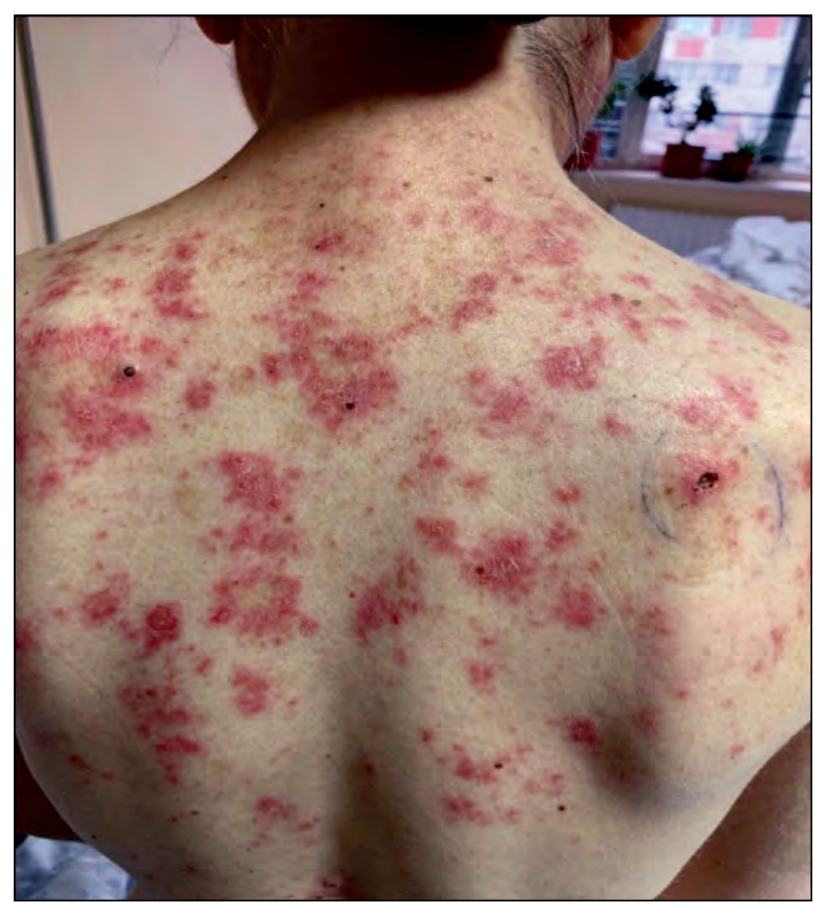

FIGURE 1 a, b. Papulo-squamous skin lesions 


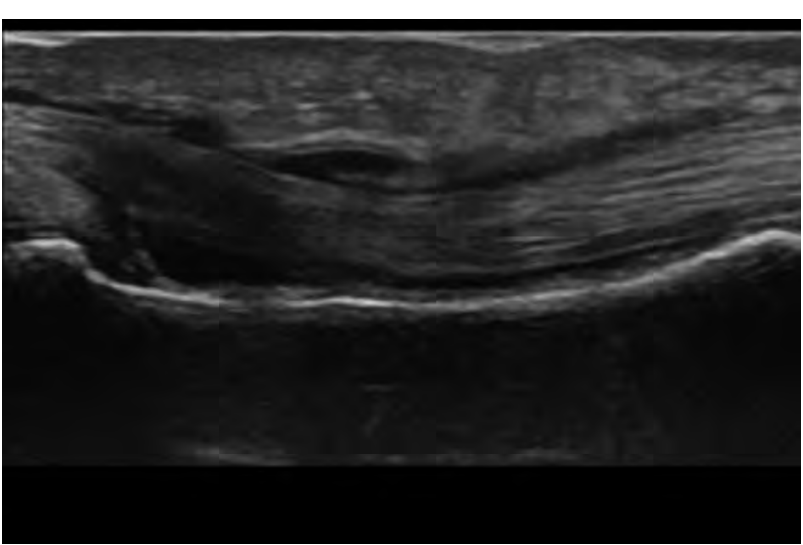

FIGURE 2. Ultrasound of the hands showing bilateral extensor tenosynovitis

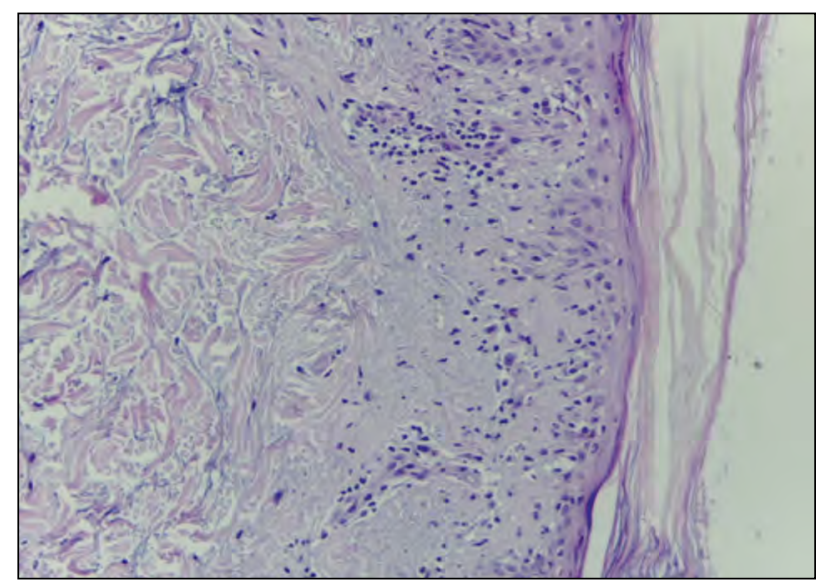

FIGURE 3. Histopathological findings showing vacuolar alteration of the basal keratinocytes and perivascular lymphoplasmacytic inflammatory infiltrate

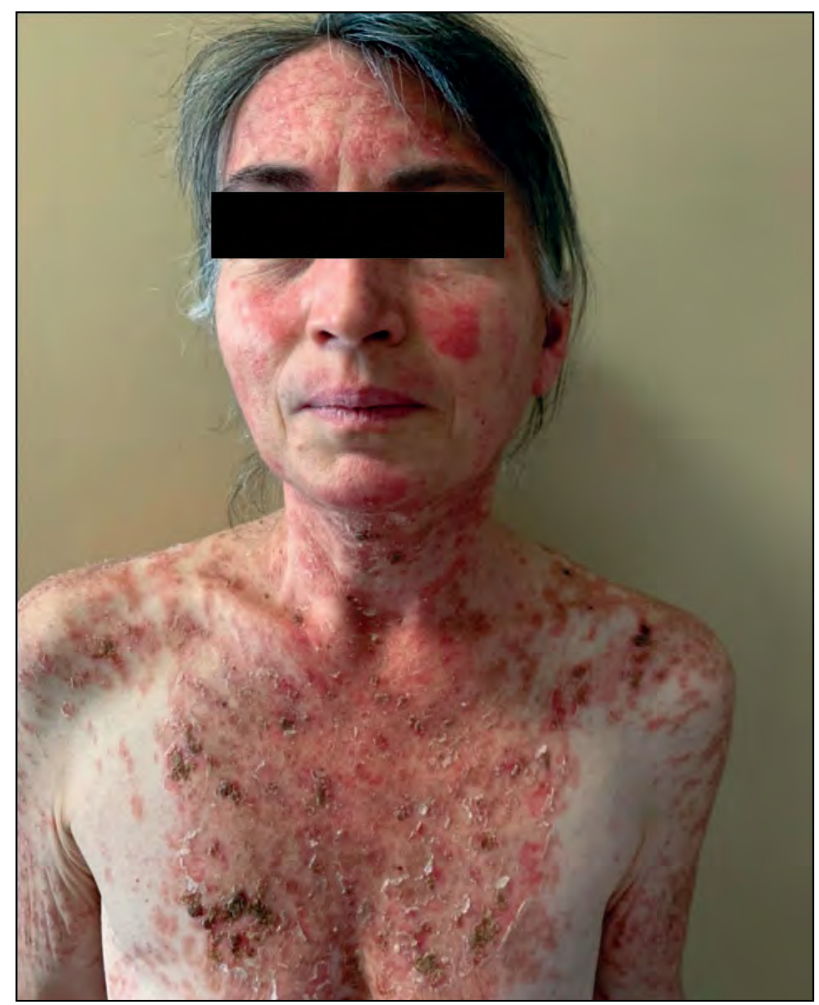

ferent from her "baseline" since diagnosis. Musculoskeletal ultrasonography of the hands and wrist revealed tenosynovitis of the extensor tendons.

Dermatology was consulted and suspected a drug-related reaction rather than SLE flare. Another skin biopsy was perfomed, revealing epidermal necrolysis with detachment and keratinocyte apoptosis in the basal layer of the epidermis (Fig. 5a, b) suggestive for toxic epidermal necrolysis (TEN). Anamnestic, clinical and paraclinical findings were consistent with TEN.

Belimumab was immediately stopped and the patient was treated with pulse IV methylprednisolone therapy $500 \mathrm{mg}$ for three consecutive days associated with gastric protection and potassium and vitamin D supplementation to prevent corticosteroids induced adverse events.. In the following three days, a progressive improvement of the skin reaction was noted. She was discharged a week later, with markedly improved aspect of the skin lesions (Fig. 6a, b). SLE treatment was changed to azathioprine $100 \mathrm{mg}$ / day and a tapering medium dose (25 mg daily) of oral corticosteroids. This adverse event was reported to the Romanian National Medications Association.

Laboratory findings showed satisfactory laboratory tests improvement (resolution hemolytic anemia and lymphopenia), being present only hypocomplementemia.This time, the dermatologist stated

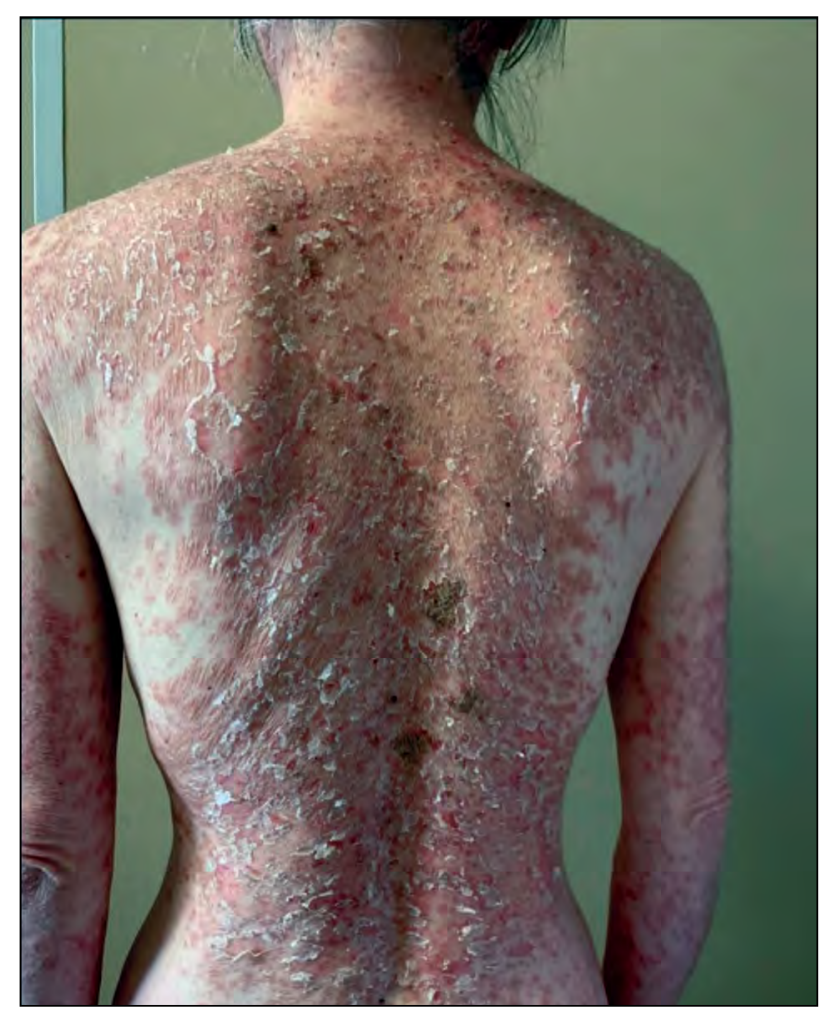

FIGURE 4 a, b. Erythemato-bullous lesions with tendency to rapid coalescence and epidermal detachment 

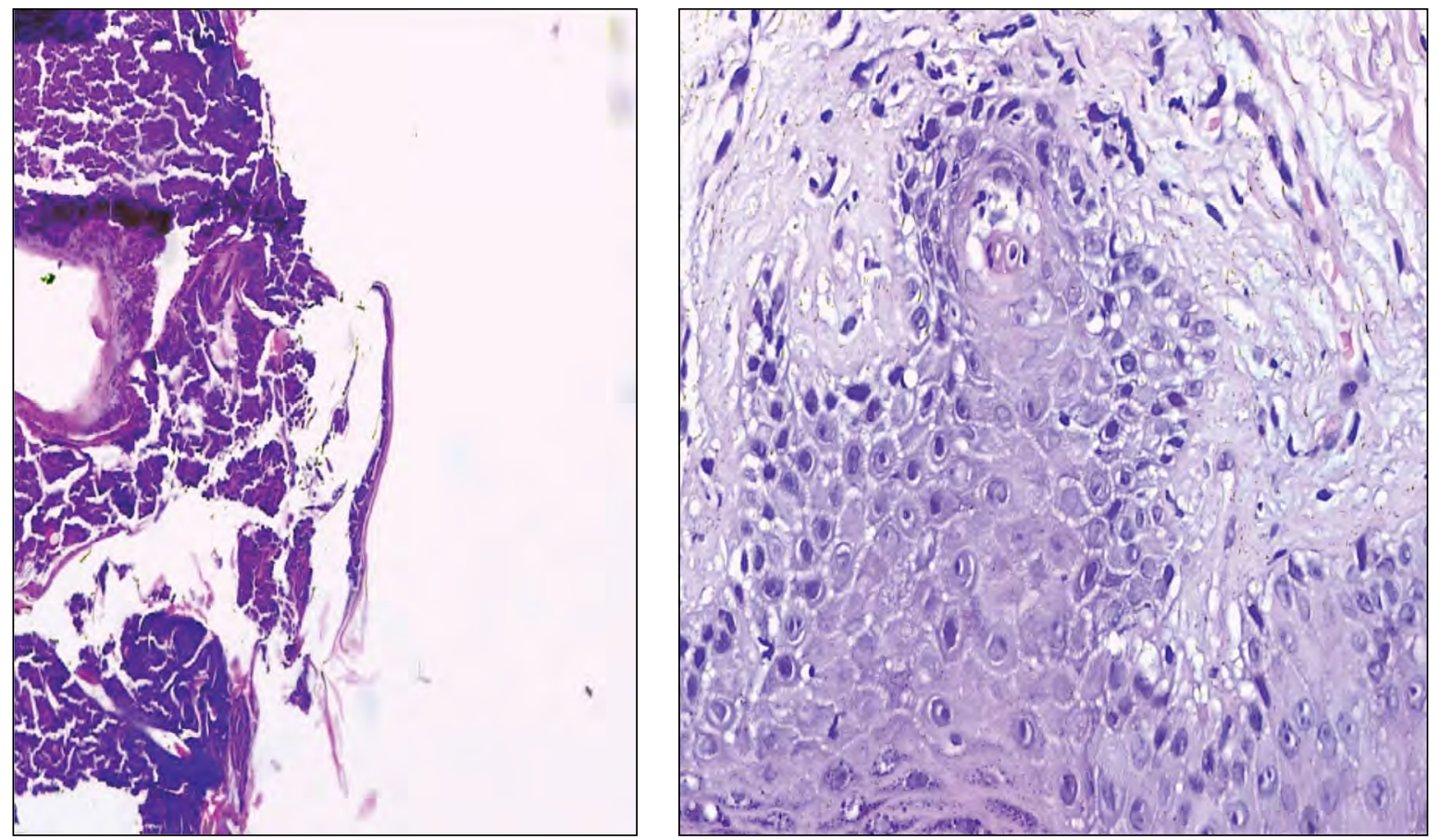

FIGURE 5 a, b. Histopathological findings showing epidermal necrolysis with detachment and keratinocyte apoptosis in the basal layer of the epidermis

for the adverse reaction to belimumab. Another skin biopsy was perfomed and it showed epidermal necrolysis with detachment and keratinocyte apoptosis in the basal layer of the epidermis (Fig. 5a, b) suggestive for toxic epidermal necrolysis (TEN).

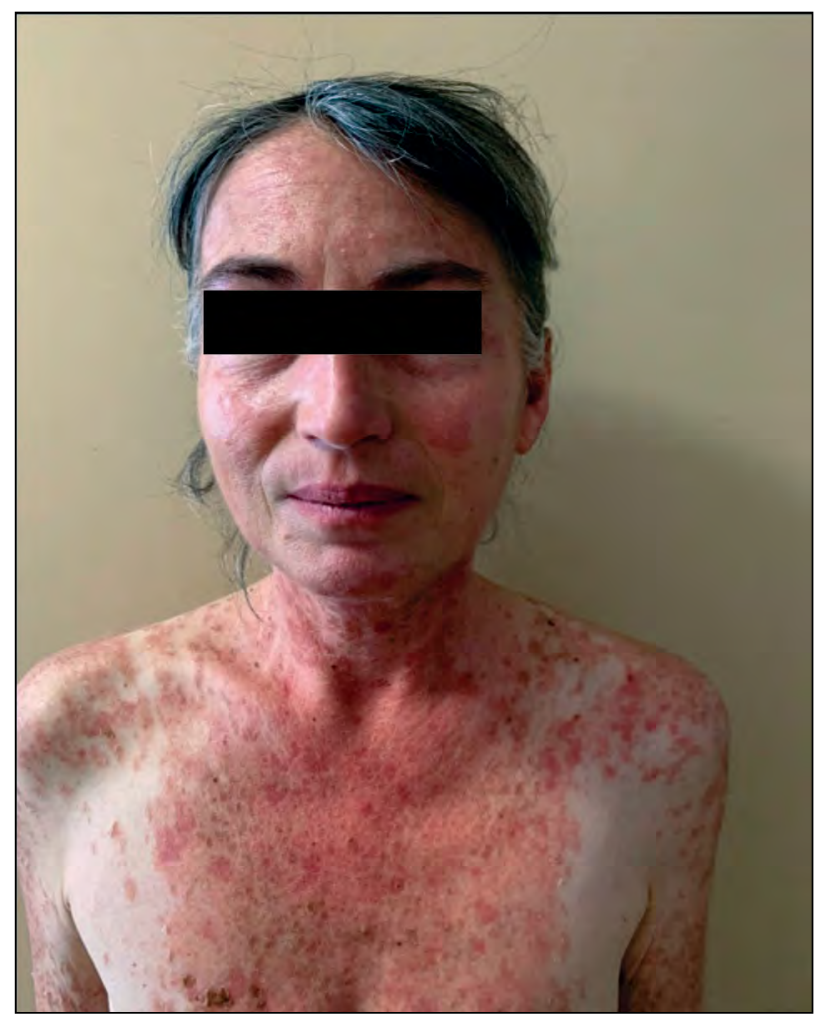

TEN is a potentially life-threatening dermatologic disorder characterized by widespread erythema, necrosis, and bullous detachment of the epidermis and mucous membranes, resulting in exfoliation and possible sepsis and/or death (7). The average report-

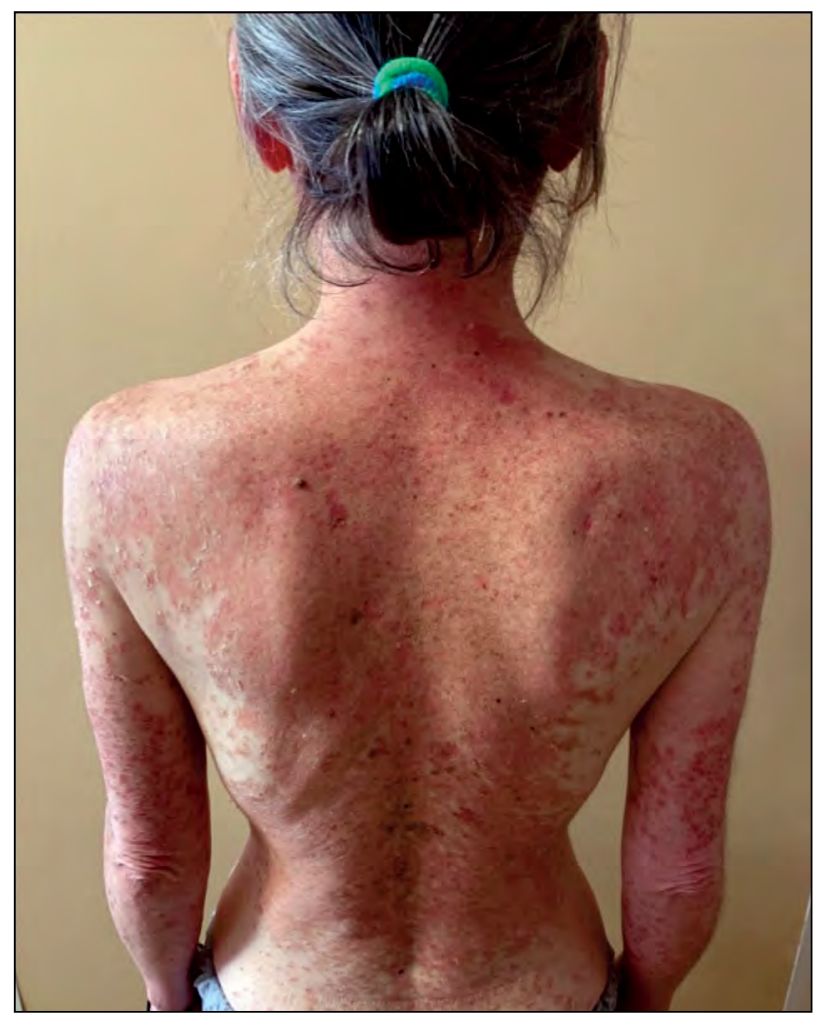

FIGURE 6 a,b. Improvement of skin lesions after treatment and hospital discharge 
ed mortality rate of TEN is $25-35 \%$; it can be even higher in elderly patients and those with a large surface area of epidermal detachment (8). A serious cutaneous reaction is more likely to occur during the first 2 months of treatment (9).

The management concentrate on resuscitative and supportive strategies with the primary concern that of avoiding infection. The general measures include immediate withdrawal of all the suspected drugs is the key to the management of TEN (10).

\section{DISCUSSION}

TEN is a potentially life-threatening dermatologic disorder characterized by widespread erythema, necrosis, and bullous detachment of the epidermis and mucous membranes, resulting in exfoliation and possible sepsis and/or death (7). The average reported mortality of TEN is $25-35 \%$; it can be even higher in elderly patients and those with a large surface area of epidermal detachment (8). A serious cutaneous reaction is more likely to occur during the first 2 months of treatment (9). Management focuses on resuscitative and supportive strategies, with the primary goal of avoiding infection. General measures include immediate withdrawal of all suspected causative drugs is the key to the management of TEN (10). TEN occurs mostly after use of medications

\section{REFERENCES}

1. Rees $F$, Doherty $M$, Grainge $M$ et al. The incidence and prevalence of systemic lupus erythematosus in the UK, 1999-2012. Annals of the Rheumatic Diseases 2016;75:136-141.

2. Dall'Era M. Systemic lupus erythematosus. In: Imboden JB, Hellman DB, Stone JH. (Eds). Current Rheumatology Diagnosis and Treatment. $3^{\text {rd }}$ ed. New York, NY:McGraw-Hill; 2013.

3. Boyce EG, Fusco BE. Belimumab: Review of use in systemic lupus erythematosus. Clin Ther. 2012 May; 34(5):1006-22. Epub 2012 Mar 30.

4. Dubey AK, Handu SS, Dubey S, Sharma P, Sharma KK, Ahmed QM. Belimumab: First targeted biological treatment for systemic lupus erythematosus. J Pharmacol Pharmacother. 2011 Oct; 2(4):317-9.

5. Vilas-Boas A, Morais SA, Isenberg DA. Belimumab in systemic lupus erythematosus. RMD Open. 2015; 1(1):e000011.

6. Specchia ML, de Waure C, Gualano MR, Doria A, Turchetti G, Pippo L, Di Nardo F, Capizzi S, Cadeddu C, Kheiraoui F, laccarino L, Pierotti F, Palla I, Veneziano MA, Gliubizzi D, Sferrazza A, Nicolotti N, Porcasi R, La Torre G, Di Pietro ML, such as anticonvulsants, neuroleptics, antibiotics, antifungals, diuretics, non-hormonal anti-inflammatories, and analgesics.

Regarding skin reactions after belimumab, data from the clinical trials and long-term follow-up studies indicate that belimumab is generally well-tolerated (11). The rate of adverse events noted in various studies varies from $6 \%$ to $38 \%$. The most common skin adverse effects include rash, cellulite.

\section{CONCLUSION}

To the best of our knowledge, this is the first report of TEN triggered by treatment with belimumab in a patient with SLE. Although rare, physicians should be aware of the possible skin reactions associated with novel biologic agents in SLE, such as belimumab.

\section{Compliance with ethical standards}

Ethical approval. All procedures performed in studies involving human participants were in accordance with the ethical standards of the institutional and/or national research committee and with the 1964 Helsinki Declaration and its later amendments or comparable ethical standards.

Informed consent. Informed consent was obtained from the patient presented in this work.

Conflict of interest: none declared Financial support: none declared

Ricciardi W. Health technology assessment of belimumab: a new monoclonal antibody for the treatment of systemic lupus erythematosus. Biomed Res Int. 2014; 2014():704207.

7. Tiwari P, Panik R, Bhattacharya A, Ahirwar D, Chandy A. Toxic epidermal necrolysis: An update. Asian Pac J Trop Dis. 2013; 3(2):85-92. doi:10.1016/S2222-1808(13)60051-1

8. Roujeau JC, Stern RS. Severe adverse cutaneous reactions to drugs. N Engl J Med. 1994 Nov 10; 331(19):1272-85.

9. Barvaliya MJ, Patel MK, Patel TK, Tripathi CB. Toxic epidermal necrolysis due to lamotrigine in a pediatric patient. J Pharmacol Pharmacother. 2012;3(4):336-338. doi:10.4103/0976-500X.103695

10. Tiwari, Prashant et al. Toxic epidermal necrolysis: An update. Asian Pacific Journal of Tropical Disease vol. 3,2 (2013): 85-92. doi:10.1016/S2222-1808(13)60051-1

11. Borchers AT, Lee JL, Naguwa SM, Cheema GS, Gershwin ME. Stevens-Johnson syndrome and toxic epidermal necrolysis. Autoimmun Rev. 2008;7:598-605. (PubMed) (Google Scholar) (Ref list) 